\title{
Empirical studies about inhibiting proliferation of zedoary turmeric oil, Cinobufacini and Solanine on gastric carcinoma cells
}

\author{
Xuejun Zhan ${ }^{1}$, Daze Xie ${ }^{1}$, Yingyin $\mathrm{Hu}^{2}, \mathrm{Ge} \mathrm{Dai}^{3}$, Yanping Xu${ }^{1}$ \\ ${ }^{1}$ Department of Detection Center, Key Laboratory of Biological Medical of Jiangxi, Jiangxi Academy of Medical Science, Jiangxi, \\ Nanchang, China \\ ${ }^{2}$ Cancer Research Laboratory, Key Laboratory of Biological Medical of Jiangxi, Jiangxi Academy of Medical Science, Jiangxi, Nanchang, \\ China \\ ${ }^{3}$ Department of Nuclear Medicine, Jiangxi Academy of Medical Science, Jiangxi, Nanchang, China
}

\section{Email address:}

zhan_xue_jun@163.com (Xuejun Zhan)

\section{To cite this article:}

Xuejun Zhan, Daze Xie, Yingyin Hu, Ge Dai, Yanping Xu . Empirical Studies about Inhibiting Proliferation of Zedoary Turmeric oil, Cinobufacini and Solanine on Gastric Carcinoma Cells. Cancer Research Journal. Vol. 2, No. 3, 2014, pp. 42-46.

doi: 10.11648/j.crj.20140203.11

\begin{abstract}
Purpose: To investigate the anti-proliferative effects of anti-cancer Chinese medicinal materials such as zedoary turmeric oil, cinobufacini and solanine on gastric carcinoma cell line NKM45. Methods: Bioluminescence assay (BLA) was applied to determine the ATP concentration when NKM45 was exposed to the three Chinese medicinal materials. The influence of zedoary turmeric oil on the growth curve of NKM45 cells was also analyzed. And the concentration of ATP was compared with NKM45 cells that were exposed to 5-Fluorouracil(5-Fu). Results: The concentration of ATP was significantly decreased when the NKM45 cells were exposed to the three Chinese medicinal materials and 5-Fu $(\mathrm{P}<0.05 \sim \mathrm{P}<0.01)$. There was no significant difference in the ATP concentration between MKM45 cells that were treated with the three Chinese medicinal materials and those exposed to 5-Fu ( $P>0.05)$. The zedoary turmeric oil significantly inhibited the survival rate of NKM45 cells. Conclusions: Zedoary turmeric oil, cinobufacin, and solanine can inhibit the proliferative activity of NKM45 cells. They have exploitation potential with broad market prospect.
\end{abstract}

Keywords: Gastric Carcinoma Cells, Anti- Cancer Chinese Medicinal Materials, ATP Concentration

\section{Introduction}

Zedoary, Bufo bufo gargarizans Cantor and solanum nigrumL.(SNL) are traditional Chinese medicinal materials and are believed to have anti-disease properties. Zedoary turmeric oil(ZTO) is a volatile oil that is extracted from zedoary. Its main elements are sesquiterpenoids including curcumenol, curdione,elemene and so on ${ }^{[1]}$. ZTO has some pharmacological actions such as hepatoprotective, to enhance blood cells, anti-embolism,antibiosis anti-virus effects $^{[2,3]}$.

Cinobufacini is a preparation of water soluble that is prepared from Bufo bufo gargarizans Cantor. It has been confirmed that cinobufacini has functions of anti-tumor, anti-virus, anti-inflammation and regulation organism immunity function ${ }^{[4]}$.

Solanine is a alkaloid that is extracted from the whole plant or unripe fruit of $\mathrm{SNL}^{[5]}$. It has sharp effects of anti-nuclear fission and cytotoxicity ${ }^{[6,7]}$.

Above the three Chinese medicinal materials have properties of wide anti-cancer spectrum and little side-toxicity effects. In recent years, either single drug or compound and extracted from them have attracted attentions in the mechanism involved inhibitory effects on tumor cells growth ${ }^{[8,9,10] \text {. }}$

Endogenous adenosine triphosphate (ATP) is energy resources of living cells. Detection of ATP contents is able to show activity degrees of cellular metabolism and to reflect cellular proliferation activity indirectly ${ }^{[11]}$ At recent bioluminescence assay (BLA) technique has been applied to analyze cellular proliferation activity, cytotoxicity and the sensitivity of chemotherapeutic agents on tumor cells ${ }^{[12,13]}$. In this study the percentage of ATP decreased was assayed after the three Chinese medicinal materials were exposed to gastric carcinoma cell line NKM45 by 
BLA method, in order to explore the inhibition proliferative effects of zedoary turmeric coil (ZTO), cinobufacini and solanine on NKM45 cells.

\section{Materials and Methods}

\subsection{Materials}

\subsubsection{The Tumor Cells Culture}

Gastric carcinoma cell line NKM45 was gifted from Professor KengYuan of Jiangxi Academy of Medical Science, China NKM45 cells were subcultured in PRMI 1640 medium with $10 \%$ fetal cal serum, penicilin $1.0 \times 10^{5} \mathrm{U} / \mathrm{L}$, streptomycin $100 \mathrm{mg} / \mathrm{L}$ and maintained in a $5 \% \mathrm{CO}_{2}$ atmosphere at $37^{\circ} \mathrm{C}$ with $95 \%$ humidity after the cells recovered. The cells were divided bottles subcultured after $0.25 \%$ trypsinization when the cells grew well.

\subsubsection{Reagents and Instruments}

PRMI 1640 medium was purchased from Hyclone U.S.A.; ATP-BLA assay kit (containing fluorescein-luciferase compound, ATP standard) from Shanghai Institute of plant physiology; 24-wells tissue culture plate from JET Biochemicals Int'I., Inc. (Canada); ZTO from Changzheng Fumin Pharmaceutical Co.Ltd.(China); Cinobufacini from Jinchan Biochem Co.Ltd(China); Solanine was prepared by referenced to the literature $^{[14]}$; 5-Fu from Jinyao amino acid Co.Ltd (China).

Biochemistry luminometer FT-732 Model from Beijing (China).

\subsection{Methods}

\subsubsection{Preparation of ATP Standard Curve}

The ATP standard was diluted to $5 \times 10^{-8} \sim 5 \times 10^{-12} \mathrm{~mole} / \mathrm{ml}$ with phosphate buffered (PBS) in turn. The luminous intensity of ATP standard of the five concentrations was determined respectively according to the kit instruction. The ATP standard curve was drawn according to logarithms of ATP concentration as abscissa and logarithms of luminous intensity pulse values as ordinate.

\subsubsection{Correction Analysis between ATP Concentration and NKM45 Cell Number}

NKM45 cells of the logarithmic growth phase were diluted to $10^{6}, 10^{5}, 10^{4}, 10^{3}, 10^{2} / \mathrm{ml}$ in turn. Two parallel tubes were designed every concentration. $4 \%$ trichloroacetic acid (TDA) of equivalent was added to every tube in order to extract ATP ${ }^{[15]}$. Then $100 \mu \mathrm{L}$ extracts were taken to be measured.

\subsubsection{Detection ATP Concentration of the Drugs on NKM45 Cells Using BLAAssay}

NKM45 cells were adjusted to $1.0 \times 10^{5} / \mathrm{ml}, 0.5 \mathrm{ml}$ cells and $0.5 \mathrm{ml}$ medium were inoculated in 24 -well plate and maintained in a $5 \% \mathrm{CO}_{2}$ atmosphere at $37^{\circ} \mathrm{C}$ with $95 \%$ humidity. When the cells grew well, five groups were assigned.

(1). ZTO group: $\mathrm{ZTO}(0.4 \mathrm{~g} / \mathrm{L}) 1.0 \mathrm{ml}$ was treated in each bore.

(2).Cinobufacini group: Cinobufacini $(0.5 \mathrm{~g} / \mathrm{L}) 1.0 \mathrm{ml}$ was treated in each bore.

(3).Solanine group: Solanine $(2.0 \mathrm{~g} / \mathrm{L}) 1.0 \mathrm{ml}$ was treated in each bore.

(4).5-Fu group: $5-\mathrm{Fu}(0.1 \mathrm{~g} / \mathrm{L}) 1.0 \mathrm{ml}$ was treated in each bore.

(5).Control group: PRMI 1640 medium 1.0ml was treated in each bore.

Two parallel bores were designed above every group. After the five group cells were incubated for $5 \mathrm{~d}$, the cellular ATP was extracted ${ }^{[15]}$.

\subsubsection{Effect of ATP Concentration of NKM45 Cells Exposed to the Drugs at Different Culture Time}

$1.0 \mathrm{ml}$ NKM45 cells suspension $\left(1.0 \times 10^{5} / \mathrm{ml}\right)$ was added to ZTO, cinobufacini, solanine and $5-\mathrm{Fu}$, the final concentration of them was $0.08,0.10,0.40$ and $0.02 \mathrm{~g} / \mathrm{L}$, respectively. The control group was treated with equivalent PRMI 1640 medium. After culturing for 24h, 48h, 72h, cellular ATP was extracted and to determine ATP concentration using BLA assay. Degradation rate of ATP was counted at different time.

Rate of ATP degradation $(\%)=1-\frac{\text { Drug }- \text { group }- \text { value }}{\text { Control }- \text { group }- \text { value }} \times 100 \%$

\subsubsection{Analysis of Luminous Intensity}

$200 \mu \mathrm{L}$ 0.05M Tris-HCl (PH9.1) was added to the tubes that contain ATP measured. According to the kit instruction, the luminous intensity of every tube was determined with the Biochemistry Luminometer. Then ATP concentration was counted in accordance with the ATP standard curve.

\subsubsection{Determination about the Influence of ZTO on Growth Curve of NKM45 Cells}

Reference to methods of Xu RC ${ }^{[16]}$, NKM45 cells in the logarithmic growth phase were adjusted to $2.0 \times 10^{4} / \mathrm{ml}$. The cells were incubated in PRMI1640 medium for 24 hours, and the medium was discarded after the cells were adherent. The ZTO was added to the medium to a final concentration of $0.1 \mathrm{~g} / \mathrm{L}$ and $0.2 \mathrm{~g} / \mathrm{L}$, respectively. The equivalent medium was added to the control group. NKM45 cells of the ZTO group and the control group were incubated for $5 \mathrm{~d}$, respectively. Everyday two bottle cells were taken from every group and the living cells were counted with trypanblue dye exclusion test. The cells growth curve was drawn according to the living cells average number. At the same time, ATP in NKM45 cells of the control group and the ZTO group were extracted and the bioluminescence intensity was determined.

\subsubsection{Statistical Analysis}

The significance of difference in ATP concentration between the control group and the drugs group was analyzed by the average $t$-test using the SPSS11.5 software. A value of $\mathrm{P}<0.05$ was considered significant. 


\section{Results}

\subsection{ATP Standard Curve}

There was positive liner correlation between the logarithms of ATP concentration and the logarithms of luminous intensity values when ATP at concentration of $5 \times 10^{-8} \sim 5 \times 10^{-12} \mathrm{~mole} / \mathrm{ml}$ and correlative coefficient $\mathrm{r}=0.936$ $(\mathrm{P}<0.01)$. Equation of a straight line was $\log Y=0.306 \log x \mid 4.59(b=0.306, P<0.05)$.

\subsection{Correlation Analysis between ATP Concentration and the Number of NKM45 Cells}

A good correlation was found between the logarithm of ATP concentration and the logarithms of NKM45 cell numbers and $\mathrm{r}=0.947(\mathrm{P}<0.01)$. Equation of a straight line was $\log Y=0.144 \log x+1.06(b=0.144, \mathrm{P}<0.05)$

\subsection{Analysis of the Drugs for Inhibiting Proliferation of NKM45 Cells}

ATP concentration was analyzed when NKM45 cells were exposed to ZTO, cinobufacini and solanine, and compared with NKM45 cells that were treated with 5-Fu according to ATP standard curve. It was found that ATP concentration was significant decreased when NKM45 cells exposed to the three Chinese medicinal materials $(\mathrm{P}<0.05, \mathrm{P}<0.05, \mathrm{P}<0.01)$ (Table 1). The percentage of ATP decrease was $94.62 \%$, $95.59 \%$, and $95 \%$, respectively. There was no significant difference of ATP concentration between the three Chinese medicinal materials and 5-Fu when they were exposed to NKM45 cells, respectively $(\mathrm{P}>0.05)$. There was also no obvious difference among ATP concentration after NKM45 cells were treated with the three drugs $(\mathrm{P}>0.05)$.

Table 1. Comparison of ATP concentration in NKM45 cells between control group and drugs group.

\begin{tabular}{ll}
\hline & ATP concentration (mole/ml) \\
& $\overline{\mathrm{X}} \pm \mathrm{s}$ \\
\hline Control group & $10^{-9}(2.34 \pm 0.88)$ \\
ZTO group & $10^{-11}(1.26 \pm 1.10)^{* *}$ \\
Cinobufaini group & $10^{-11}(9.66 \pm 1.57)^{* *}$ \\
Solanine group & $10^{-10}(1.17 \pm 0.84)^{*}$ \\
5-Fu group & $10^{-11}(1.22 \pm 0.47)^{* *}$ \\
\hline
\end{tabular}

Drug group $v s$ control group ( $t$-test $): * \mathrm{P}<0.05, * * \mathrm{P}<0.01$

\subsection{Relation of between ATP Concentration and the Drugs with NKM45 Cells at Different Culture Time}

The results showed that the percentage of ATP concentration degradation in $72 \mathrm{~h}$ was obvious higher than in 24h (t-test, $\mathrm{P}<0.01$ ) (Table 2)
Table 2. Percentage of ATP degradation of NKM45 cells with the drugs at different culture time $(\bar{x} \pm S) \%$.

\begin{tabular}{lllll}
\hline $\begin{array}{l}\text { Culture } \\
\text { time }\end{array}$ & ZTO & Cinbufacini & Solannine & 5-Fu \\
\hline & $0.08 \mathrm{~g} / \mathrm{L}$ & $0.1 \mathrm{~g} / \mathrm{L}$ & $0.4 \mathrm{~g} / \mathrm{L}$ & $0.02 \mathrm{~g} / \mathrm{L}$ \\
$24 \mathrm{~h}$ & $13.30 \pm 0.80$ & $11.72 \pm 1.15$ & $8.78 \pm 0.78$ & $10.33 \pm 1.50$ \\
$48 \mathrm{~h}$ & $16.05 \pm 0.32$ & $20.09 \pm 2.19$ & $17.10 \pm 1.63$ & $22.18 \pm 1.56$ \\
$72 \mathrm{~h}$ & $37.55 \pm 1.50$ & $42.09 \pm 2.22$ & $40.11 \pm 1.40$ & $45.15 \pm 1.52$ \\
\hline
\end{tabular}

\subsection{The Influence of ZTO on Growth Curve of NKM45 Cells}

ZTO $(0.1 \mathrm{~g} / \mathrm{L}, 0.2 \mathrm{~g} / \mathrm{L})$ and NKM45 cells were cultured for $5 \mathrm{~d}$, continuously. The living cells were counted every day. It was found that there was inhibiting effects in ZTO of the two concentrations on the growth of NKM45 cells (Fig3). The survival rates of the cells in drug group of high concentration obvious lower than the drug group of low concentration with prolonged time exposure.

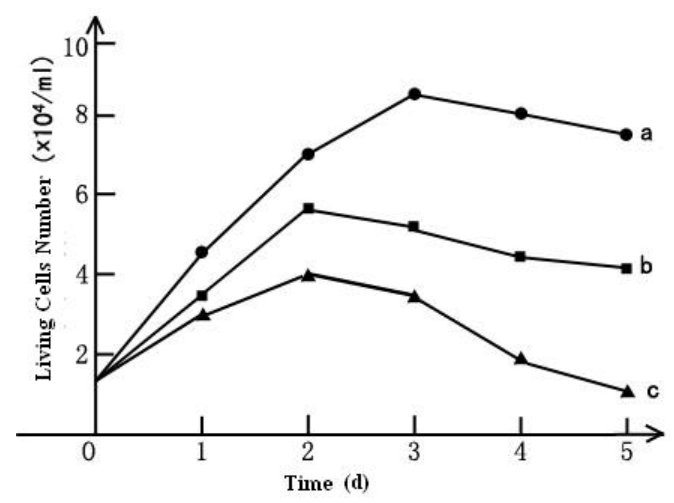

Fig 1. Growth curve of NKM45 cells. a. Control group b. ZTO group $(0.1 \mathrm{~g} / \mathrm{L})$ c. ZTO group $(0.2 \mathrm{~g} / \mathrm{L})$.

\section{Discussion}

A content of ATP intracellular is relative constant in physiologic state. When cellular metabolism is impaired by drugs, ATP synthesize is obstructed and cells death and ATP is disappeared. ATP content of assay is not only to detect cellular death, but is also able to evaluate cellular activity $^{[17]}$. A measurable light can be produced when ATP intracellular was exposed to containing fluoresceinluciferase compound. In this study the first intracellular ATP was released by splitting action of trichloroacetic acid and then fluorescein-luciferase compound was added. Fluorescence was produced by the chemical reaction between fluorescein-luciferase compound and ATP. By means of biochemistry luminometer, fluorescence intensity that is able to reflect ATP content to be measured samples is detected. Thus number of living cells can be reflected. To explore inhibition effect of ZTO, cinobuficini and solanine on NKM45 cells, the authors have analyzed correlation between ATP concentration and luminous intensity; cellular concentration and luminous intensity in the experiment by 
BLA method. Effect of ZTO on the growth curve of NKM45 cells and ATP concentration were also analyzed when NKM45 cells were treated with the three Chinese medicinal materials. The research results showed that there were positive correlation between ATP concentration and correspondent luminous intensity, the cellular concentration and correspondent luminous intensity. Comparing with the control group ATP concentration was significantly decline when NKM45 cells were treated with the three drugs. There was no significant difference in response between ATP concentration of NKM45 cells that were treated with the three Chinese medicinal materials and those exposed to 5-Fu. There were obvious inhibiting effects of survival rate of ZTO $(0.1 \mathrm{~g} / \mathrm{L}, 0.2 \mathrm{~g} / \mathrm{L})$ on NKM45 cells and the positive correlation between ATP concentration and the living cells number resembling the result of Sevin BU et al ${ }^{[18]}$.

By Chinese medicinal materials or combined methods of treatment of traditional Chinese and Western medicine, a malignant tumor is cured effectively ${ }^{[19]}$. The treatment methods enable to decrease toxicity and side-effects of the radiotherapy and chemotherapy, to increase tolerance of tumor patients to the treatment, thereby improvement therapeutic efficacy. ZTO, cinobufacini and solanine that were elected in this research are anti-cancer Chinese medicinal materials. They have the effects of direct inhibition and destruction to tumor cells. It is reported that ZTO and its preparation have obvious inhibition to growth of mouse Ehrlich Ascites Carcinoma (EAC), ascites hepatoma cells, endometrial carcinoma cell and various tumor cell lines in vitro ${ }^{[1,20]}$. The main activity element of ZTO anti-cancer, curcumol and $\beta$-elemene have direct cytotoxic effect on L1615 leukemic cell ${ }^{[21]}$. Besides, zedoary is able to increase immunogenicity of tumor cell and to promote the immunologic reaction of the organism to tumor cells ${ }^{[1]}$. Cinbufacini was able to induce apoptosis in gastric cancer cell line MGC-803 through to activate the pathway of PI3K/AKt and to enhance activity of Caspase-3 ${ }^{[22]}$. It has well curative efficacy for gastric carcinoma, primary hepatoma, esophagus cancer and lung cancer. The single drug is both used to cure of malignant tumor and to combine with radiotherapy and chemotherapy in order to exert its synergist and attenuated toxicity effect ${ }^{[23]}$. Solanine can inhibit growth of MCF-7 cells and to induce apoptosis of the tumor cells ${ }^{[24,25]}$. It has also inhibition effects for mouse cervical cancer, EAC and sarcoma180 cells and to prolong the living period of mouse solid tumor ${ }^{[8,26]}$. These Chinese medicinal materials have been extensively applied in clinic and have exploitation potential with broad market prospect $^{[27,28,29]}$.

\section{Acknowledgements}

The authors thank Cancer Research Laboratory of Jiangxi Academy of Medical Science for technical assistance. This study was supported by a grant from the Health Department of Jiangxi Province for Science Technical program Foundation (No.0303072).

\section{Conflict of Interest}

The authors have no potential conflicts of interest.

\section{References}

[1] Zhou H, Wang Q, Jiang Zh Sh. Advance in anti-tumor of zedoary turmeric oil. Tumor Journal of World.2003; 2 (4) :325-328.

[2] Yang F Q, L i S P, Chen Y, et al Identification and quantitation of eleven sesquiterpenes in three species of Curcuma rhizomes by pressurized liquid extraction and gas chromatography-mass spectrometry. Journal of Pharmaceutical and Biomedical Analysis. 2006;39 (3-4): 662-668. DOI:10.1016/j.jpbc.2005.05.001

[3] You J, Cui F D, H an X, et al. Study of the preparation of sustained-release microspheres containing zedoary turmeric oil by the emulsion-solvent-diffusion method and evaluation of the self-emulsification and bioavailability of the oil. Colloids and Surfaces B: Biointerfaces.2006;48 (1): 35-41. DOI:10.1016/j.colsurfb.2005.12.011

[4] Zhang BL, Wu H. Study progress on anti-tumor and its mechanism of cinobufagini. Chinese and Foreign Medical Research.2012; 10 (14):152-154

[5] Gui XL. Mechanism research advance in anti-tumor of solanine. Chinese Journal of Information on Traditional Chinese Medicine.2009;16(suppl):80-82

[6] Zhao X Q, Zeng X F. Clinical study of Longkui tablet on primary carcinoma of the liver. Liaoning Traditional Chinese Medical.2002;29(11); 671-672

[7] JasonM Walvaven, JohnOTrent, DavidW. Hein. Structurefunction analyses of single nucleotide polymorphism in humanN-acetyltransferase1. Drug Metabolism Reviews, 2008;40(1):169-184. DOI:10.1080/03602530701852917

[8] Liu WW, Liu YQ, Dai XJ. Study advance on anti-cancer effects of Solanum NigrumL..Journal of Chinese Medicinal Materials. 2009;32(3): 462-465

[9] Qi F, Li A, Inagaki Y, etal. Antitumor activity of extracts and compounds from the skin of the toad Bufobufo gargarizans Cantor. International Immunopharmacogogy.2011;11(3):342-349. DOI:10.1016/j.intimp.2010.12.007

[10] Tang Y, Li XH. Inhibitory effects of Zedoary Rhizome abstraction hepatic cell line HepG-2 and the mechanisms. Pharmacological Bulletin. 2007; 23(6):790-794

[11] .Kangas L, Gronroos M, Nieminen AL. Bioluminescence of cellular ATP: a new method for evaluating cytotoxic agents in vitro.Medical Biology.1984;62:338- 343. PMID: 6543460

[12] Kurbacher CM, Bruckner HW, Andreotti PE, et al. In vitro activity of titanocenedichloride versus cisplatin in four ovarian carcinoma cell lines evaluated by a microtiter plate ATP bioluminescence assay. Anticancer Drugs. 1995;6(5): 697-704. PMID:8845481

[13] Crouch SP, Kozlowski R, Slater KJ, et al. The use of ATP bioluminescence as a measure of cell proliferation and cytotoxicity.Journal of ImmunologicalMethods. 1993;160 (1): 81-88. DOI:10.1016/0022-1769(93)90011-U 
[14] Chen DC. Work book of Chinese Medicine Chemical Contrasts. Chinese Medicine Scientific and Technical Press, Beijing 2000;.6-7

[15] Sevin BU, Peng ZL, Pervas JP, et al. Application on ATP-bioluminescence assay in human tumor chemosensitivity testing.GynecologicOncology.1988;31(1):191-204.DOI:1016/0 090-8258(88)9093-4

[16] Xu RC, Chen XY, Chen L, et al. Preliminary study on apoptosis of human leukemic cells induced by bufalin. China Journal of Chinese Materia Medica.2001;26:59-61. PMID:12525125

[17] Hou Y, Wu XQ, Wang XH. Research on bioluminescent theory and applying of ATP. China Medical Herald, 2010;7(12):12-18.

[18] Sevin BU, Pervas JP, Averette HE, et al. Chemosensitivity test in ginovarian cancer. Cancer. 1993; 71(4):1613-1620. DOI:10.1002/cncr2820710428

[19] $\mathrm{Xu}$ ChG. Clinical efficacy of integration in the treatment of advanced malignant tumor and development of anticancer traditional Chinese Medicine. Medical Innovation of China. 2013;10(18):141-142

DOI:10.3969/j.issn.1674-4985.2013.18.069

[20] Peng BX, He X, Shi JSh, et al. Effects of volatile oil and three main components from curcum a phaeocaulis valeton on liver cancer and endometrial carcinoma cell lines. West China Journal of Pharmaceutical Science.2007; 22(3):312-313

[21] Li GD, Xu F, Shen AJ. Studies progress of zedoary turmeric oil. Chinese Pharmaceutical Journal. 2002; 37(11):.806-809.
[22] $\mathrm{Li} \mathrm{D}, \mathrm{Qu} \mathrm{X}$, Hou K, et al. PI3K/Akt is involved in bufalin-induced apoptosis in gastric cancer cells. AnticancerDrugs.2009;20(1):59-64. DOI:10.1097/ CAD.0b013e32831160fd6

[23] Zuo XD, Cui YA. Clinical studies advance in anti-tumor of cinobufacini. Chinese Clinical Oncology. 2003; 8:232-235.

[24] Son YO, Kim J ,Lim JC, et al. Ripe fruits of Solanum nigrum L. inhibits cell growth and induces apoptosis in MCF-7 cells. Food and Chemical Toxicology.2003;41(10):1421-1428. DOI:10.1016/S0278-6915(03)00161-3

[25] Yen GC, Chen HY, Peng HH. Evaluation of the cytotoxicity, mutagenicity and anti-mutagenicity of emerging edible plants.Food and Chemical Toxicology.2001;39,(11):1045-1053. PMID:11527563

[26] Li PW. Practical clinical anti-tumor Chinese medicinal materials. Liaoning Scientific and Technical Press, Shenyang. 2000:22-23

[27] $\mathrm{Li} \mathrm{Y,} \mathrm{Lin} \mathrm{AH.} \mathrm{The} \mathrm{latest} \mathrm{research} \mathrm{progress} \mathrm{of} \mathrm{zedoary}$ turmeric oil. China practical Medicine. 2012;7(5): 243-244

[28] Mei QX, Zhang ZhQ, Guan J, et al. Research progress in clinical application of solanum nigrumL. Asia-Pacific Traditional Medicine. 2011;7(11):168-170

[29] Wang TT, Xu GX. Advancement on study of pharmacological action and clinical application of cinobufacini. International Journal of Ophthalmolgy. 2009;9(7):1330-1333 